increase and TI. Comparison of VBD formation between groups was performed using Fisher's exact test.

Results Successful VBD induction was noted in 67\% (18 of 27) $\mathrm{C} 57 \mathrm{BL} / 6 \mathrm{~J}$ strains vs $0 \%$ (0 of 19$)$ in SV129 strains $(\mathrm{P}<0.001)$. VBD was not observed in sham-operated controls. No effect was seen as a function of duration. Both TI and diameter increase for BA were greater in the C57BL/6 J strain compared to SV129strain $(56.3 \pm 16.4$ vs $21.1 \pm 21.6$ for diameter, $17.4 \pm 7.6$ vs $10.4 \pm 3.8$ for TI). Expression of proand active MMP 2 and -9 were elevated in elastase-injected $\mathrm{C} 57 \mathrm{BL} / 6 \mathrm{~J}$ animals compared to corresponding controls as well elastase-injected SV129 animals. C57BL/6 J subjects demonstrated arterial wall remodeling characterized by internal elastic lamina disruption, muscular layer discontinuity, inflammatory cell infiltration and high matrix metalloproteinases expression in the media.

Conclusion C57BL/6 J mice demonstrated greater susceptibility to VBD induction than SV129 mice.

Disclosures Y. Zhu: None. H. Xing: None. D. Dai: None. D. Kallmes: None. R. Kadirvel: None.

\section{0-039 COMPARISON OF THE MEDIUM-TERM OUTCOME OF TWO DIFFERENT METHODS FOR THE CEMENT AUGMENTATION OF INSUFFICIENCY FRACTURES OF THE SACRUM}

${ }^{1} \mathbf{R}$ Andresen, ${ }^{2} \mathrm{~S}$ Radmer, ${ }^{3} \mathrm{~J}$ Andresen, ${ }^{4} \mathrm{H}$ Schober. ${ }^{1}$ Westkuestenklinikum Heide, Acad. Teach. Hospital of the Universities of Kiel, Luebeck and Hamburg, Institut für Diagnostische und Interventionelle Radiologie/Neuroradiologie, Heide, Germany; ${ }^{2}$ Center for Orthopaedics, Berlin, Germany; ${ }^{3}$ Werner Heisenberg high school, Heide, Germany; ${ }^{4}$ Municipal Hospital Suedstadt Rostock, Academic Teaching Hospital of the University of Rostock, Department of Internal Medicine I, Rostock, Germany

\subsection{6/neurintsurg-2016-012589.39}

Introduction The objective of this prospective, randomized study was to test the feasibility and the clinical outcome of the different forms of treatment.

Material and methods In 40 patients with a total of 57 sacral fractures, cement augmentation was performed with CT-guidance by means of balloon sacroplasty (BSP) or radiofrequency sacroplasty (RFS). For BSP, the balloon catheter was inflated and deflated in the fracture zone, and the hollow space created was then filled with PMMA cement. For RFS, a flexible osteotome was initially used to extend the spongious space in the fracture zone. The highly viscous PMMA cement, activated by radiofrequency, was then inserted into the prepared fracture zone. Pain intensity was determined on a visual analogue scale before the intervention, on the second day, and 6, 12 and 18 months after the intervention. The results were tested for significance by means of paired Wilcoxon rank-sum tests and Mann-Whitney U tests.

Results BSP and RFS were technically feasible in all patients. An average of $6.3 \mathrm{ml}$ cement per fracture were inserted in the BSP group and an average of $6.1 \mathrm{ml}$ per fracture in the RFS group. Leakage could be ruled out for both procedures. The mean pain score on the VAS before the intervention was $8.6 \pm 0.55$ in the BSP group and $8.8 \pm 0.58$ in the RFS group. On the second postoperative day, a significant pain reduction was seen $(\mathrm{p}<0.001)$, with an average value of 2.5 $(\mathrm{BSP} \pm 0.28, \mathrm{RFS} \pm 0.38)$ for both groups. After $6(12 ; 18)$ months, these values were stable for the BSP group at $2.3 \pm$ $0.27(2.3 \pm 0.24 ; 2.0 \pm 0.34)$ and for the RFS group at $2.4 \pm 0.34(2.2 \pm 0.26 ; 2.0 \pm 0.31)$. With regard to pain, exceedance probability values of $\mathrm{p}=0.86$ (6 months) and $\mathrm{p}=1$ (18 months) were seen, so that neither treatment method leads to differences in results.

Conclusion BSP and RFS are interventional, minimally invasive procedures that enable reliable cement augmentation and achieve equally good clinical outcomes in the medium term.

Disclosures R. Andresen: None. S. Radmer: None. J. Andresen: None. H. Schober: None.

\section{0-040 WOVEN ENDOBRIDGE DEVICE IN TREATMENT OF ENDOVASCULAR ANEURYSMS: A META-ANALYSIS}

${ }^{1}$ W Brinjikji, 'S Asnafi, ' $A$ Rouchaud, ${ }^{2} \mathrm{~L}$ Pierot, ${ }^{1} \mathrm{D}$ Kallmes. 'Radiology, Mayo Clinic, Rochester, MN; ${ }^{2}$ Radiology, Hôpital Maison Blanche, REIMS, France

\subsection{6/neurintsurg-2016-012589.40}

Background and purpose We performed a meta-analysis of existing literature on the Woven EndoBridge (WEB) device in treatment of intracranial aneurysms.

Method A comprehensive literature search of the databases PubMed, Ovid MEDLINE and Ovid EMBASE was designed and conducted by an experienced librarian with input from the authors. We extracted the information including aneurysm rupture status, mean aneurysm size, mean aneurysm neck size, length of clinical follow-up, and length of angiographic follow-up. Outcomes studied included immediate and long-term (>3 month) angiographic outcomes (complete occlusion, as well as adequate occlusion defined as complete occlusion or neck remnant), aneurysm retreatment, intraoperative rupture, perioperative morbidity and mortality, thromboembolic complication, and treatment failure. The meta-analysis was performed with the statistical package Comprehensive Meta-Analysis.

Results A total of 14 studies were included in this analysis, including 543 patients with 557 aneurysms, of which 127 were ruptured. Treatment failure occurred in $3 \%$ of cases $(95 \%$ confidence interval $[\mathrm{CI}], 2 \%-5 \%)$. Perioperative morbidity and mortality rates were 5\% (95\% CI, 2\%-7\%) and 1\% (95\% CI, 0\%-2\%), respectively. Initial complete occlusion rates and adequate occlusion rates were $27 \%$ (95\% CI, 15\%-39\%) and 58\% (95\% CI, 36\%-79\%), respectively. Complete occlusion and adequate occlusion rates at follow-up were 39\% (95\% CI, 26\%-52\%) and 80\% (95\% CI, 75\%-85\%), respectively.

Conclusions Early data suggests that WEB treatment has an excellent safety profile and promising rates of adequate occlusion, especially given the complexity of aneurysms treated. Further prospective clinical registries and trials are needed to confirm these results and better define the risks and benefits of the use of the WEB device in treatment wide-necked and wide-neck bifurcation aneurysms.

Disclosures W. Brinjikji: None. S. Asnafi: None. A. Rouchaud: None. L. Pierot: 1; C; Sequent. D. Kallmes: 1; C; Sequent.

\section{0-041 THE ANEURYSM SIZE AND WINDKESSEL EFFECT: A CONTRAST TRANSIT TIMES STUDY ON DIGITAL SUBTRACTION ANGIOGRAPHY}

\footnotetext{
${ }^{1} \mathrm{~A}$ Hussein, ${ }^{2} \mathrm{~A}$ Linninger, ${ }^{1} \mathrm{~F}$ Charbel, II, ${ }^{3} \mathrm{C}$ Hsu, ${ }^{1} \mathrm{~F}$ Charbel, ${ }^{1} \mathrm{~V}$ Aletich, ${ }^{1} \mathrm{~A}$ Alaraj. ${ }^{1}$ Neurosurgery, University of Illinois at Chicago, Chicago, IL; ${ }^{2}$ Neurosurgery, Bioengenerring, University of Illinois at Chicago, Chicago, IL; ${ }^{3}$ Bioengenerring, University of Illinois at Chicago, Chicago, IL
}

10.1136/neurintsurg-2016-012589.41 